$\begin{array}{cl}\begin{array}{cl}\text { Revue } \\ \text { de Ihistoire }\end{array} & \text { Revue de l'histoire des religions } \\ \text { des religions } & 4 \mid 2010 \\ & \text { Qu'est-ce qu'un « paysage religieux »? }\end{array}$

\title{
Réflexions autour de l'inventaire topographique des lieux de culte (programme CIRCE)
}

Comments on the Topographical Inventory of Cult Areas (CIRCE program)

\section{Sonia Darthou}

\section{(2) OpenEdition \\ Journals}

Édition électronique

URL : http://journals.openedition.org/rhr/7690

DOI : $10.4000 /$ rhr.7690

ISSN : 2105-2573

Éditeur

Armand Colin

Édition imprimée

Date de publication : 1 décembre 2010

Pagination : 703-712

ISBN : 978-2200-92658-8

ISSN : 0035-1423

\section{Référence électronique}

Sonia Darthou, « Réflexions autour de l'inventaire topographique des lieux de culte (programme CIRCE) », Revue de l'histoire des religions [En ligne], 4 | 2010, mis en ligne le 01 décembre 2013, consulté le 06 mai 2019. URL : http://journals.openedition.org/rhr/7690 ; DOI : 10.4000/rhr.7690 


\section{Réflexions autour de l'inventaire topographique des lieux de culte (programme CIRCE)}

L'objectif du programme est de constituer un inventaire topographique des lieux de culte connus par l'archéologie et les témoignages épigraphiques dans le monde grec et romain sous la forme d'une base de données associée à un système d'information géographique. Cette base, aujourd'hui en ligne, présente les résultats sur notre première région grecque, la Béotie, en rassemblant pour chaque lieu de culte les données qui lui sont relatives, l'ambition étant de contribuer à " reconstruire » la topographie religieuse antique et d'éclairer des thèmes de recherche actuels : la définition d'un lieu de culte, la notion de l'" occupation religieuse du territoire » ou les liens entre réalités naturelles et lieux de culte.

\section{Comments on the Topographical Inventory of Cult Areas (CIRCE program)}

This program aims to set up a topographical inventory of cult areas (known thanks to archaeological and epigraphical evidence in the Greek and Roman world) as a data base associated with a geographical information system. This data base, which currently exists online, presents the results of our first Greek region, Beotia, and provides the data of each one of its cult areas. Our ambition is to contribute to the "reconstruction" of the cult topography of antiquity and to throw new light on current research themes: defining a cult area, the notion of the "religious occupation of the territory", and the link between natural landscape and cult areas. 
L'objectif du programme CIRCE (Constructions, interprétations et représentations cultuelles de l'espace dans les sociétés anciennes $)^{1}$ soutenu par l'ANR ${ }^{2}$ est de constituer un inventaire topographique des lieux de culte connus par l'archéologie et les témoignages épigraphiques dans le monde grec et en Italie ${ }^{3}$, région par région, sous la forme d'une base de données relationnelle ${ }^{4}$ associée à un système d'information géographique. Ce projet, qui vise à constituer un «atlas numérique cultuel», découle de la prise de conscience dans les recherches récentes de l'importance du contexte topographique pour comprendre ces lieux de culte; et de la volonté de rassembler les différentes données les concernant pour recenser de manière coordonnée les structures, le mobilier trouvé en contexte, mais aussi les inscriptions liées à ces sites. Il vise à renouveler et à approfondir l'analyse des significations de la répartition des cultes, des rites et des offrandes dans l'espace.

\section{RÉFLEXIONS MÉTHODOLOGIQUES ET OBJECTIFS DU PROGRAMME}

Depuis plusieurs années, la réflexion sur la spatialité s'intensifie et l'on voit le développement d'une anthropologie de l'espace qui permet d'articuler les études de géographie historique aux travaux sur les systèmes polythéistes et sur la notion de sanctuaire. L'enjeu est de prendre en compte les processus de territorialisation, les monuments, les pratiques et les parcours rituels qui inscrivent une société dans son espace, forment son « armature religieuse » et contribuent à façonner son identité, afin d'impulser des recherches sur une « archéologie du paysage » au niveau du religieux ${ }^{5}$. L'ambi-

1. Le programme CIRCE associe les centres Louis Gernet et Gustave Glotz (qui ont fusionné dans l'UMR 8210 ANHIMA), l'École française d'Athènes et le Collège de France.

2. Programme BLANC ANR-OS-BLAN-0013-01.

3. Pour l'Italie, le programme «Fana, Templa, Delubra » est dirigé par John Scheid au Collège de France et au sein de l'UMR 8210 ANHIMA.

4. Base consultable en ligne à l'adresse http://www.circe-antique.fr, dont la conception et la réalisation ont été menées par Sonia Darthou, Jean-Sébastien Gros et Ioanna Patera.

5. L'expression «archéologie du paysage » est étudiée par Philippe Leveau dans « Archéologie, espace et environnement : des paysages aux risques naturels », 
tion du programme est ainsi d'élaborer, à partir d'une réflexion collective, un outil de référence qui puisse rassembler l'ensemble des sources dispersées dans des ouvrages ou des bases de données qui procèdent généralement par classement thématique (par divinité ou épiclèses notamment) et séparent artificiellement les champs de recherche d'un même lieu de culte ou d'un même territoire, sans les inscrire précisément dans leur espace, ni les mettre en contexte par rapport aux réalités géographiques. Le projet souhaite donc rassembler et harmoniser ces sources documentaires dans un système cartographique unifié avec une mise en contexte face aux paramètres géographiques (relief de montagne, bord de mer, rivière...) ou anthropiques (frontière, habitat, voie de circulation). Cette recension raisonnée, basée sur une logique topographique, a abouti à l'élaboration d'un outil de recherche proposant une vision renouvelée, homogène et précise qui devrait permettre des comparaisons pertinentes et amorcer une réflexion autour de l'espace religieux et de ses évolutions. Avec la possibilité essentielle de pouvoir en permanence actualiser nos données en intégrant les nouvelles trouvailles, mais aussi les récentes interprétations. Le projet porte sur les lieux de culte antiques du $\mathrm{x}^{\mathrm{e}}$ siècle avant notre ère jusqu'au $\mathrm{VI}^{\mathrm{e}}$ siècle après. La possibilité existe d'élargir cette fenêtre chronologique par des renvois antérieurs ou des évolutions postérieures, afin de présenter des situations de continuité ou d'aborder potentiellement le dossier de la christianisation du paysage religieux. Le projet est scindé pour le moment autour de trois axes géographiques - la Grèce, l'Italie et la Tunisie antiques -, qui ont vocation à être présentés de concert dans un futur proche ${ }^{6}$. Pour l'Italie, si l'inventaire a commencé dans une logique de publication ${ }^{7}$, les données sont en

dans Françoise Dumasy, François Queyrel (éd.), Archéologie et environnement dans la Méditerranée antique, Genève, Droz, 2009, p. 1-22. Pour ces questionnements, voir Olivier de Cazanove, John Scheid (éd.), Sanctuaires et sources. Les sources documentaires et leurs limites dans la description des lieux de culte. Actes de la table ronde organisée par le Collège de France, l'UMR 8585 Centre Gustave Glotz, l'École française de Rome et le Centre Jean Bérard, Naples, Centre Jean Bérard, 30 novembre 2001. Naples, 2003 (collection du Centre Jean Bérard 22).

6. Sur la plateforme Open melodie, actuellement en cours d'élaboration au Collège de France et qui sera abordée plus loin.

7. Sandra Gatti, Maria Romana Picuti, Fana, templa, delubra. Corpus dei luoghi di culto dell'Italia antica. 1. Regio I, Alatri, Anagni, Capitulum Hernicum, Ferentino, Veroli, Roma, Edizioni Quasar, 2008. Le deuxième fascicule est à paraître en 2010 : Idem, Fana, templa, delubra. Corpus dei luoghi di culto 
cours d'intégration dans la base numérique. Pour le volet grec, les résultats de l'inventaire ont été directement incorporés dans la base de données, qui renseigne pour le moment une première région, la Béotie. Quant à la Tunisie, qui est le troisième pays où l'inventaire a récemment débuté ${ }^{8}$, les résultats seront prochainement versés dans la base commune.

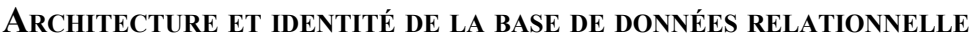

Un inventaire des lieux de culte est un programme complexe, car il peut comporter de nombreux risques d'approximations ou d'interprétations inconscientes selon la manière dont on recense, classe et affiche les données. Les champs d'une base de données ne sont pas, en effet, de simples modules de classement, ils peuvent déjà imposer une grille de lecture ou une surinterprétation. La réflexion méthodologique menée au sein du groupe CIRCE a permis de définir les sources à prendre en compte et l'architecture de la base de données relationnelle, en étroite relation avec les séminaires de François de Polignac à l'École Pratique des Hautes Études ${ }^{9}$. L'inventaire se concentre sur la prise en considération des sources archéologiques et épigraphiques afin de garder la cohérence des données et de ne pas afficher sur le même plan des sites renseignés par des vestiges, ou des attestations épigraphiques, avec des cultes connus uniquement par les sources littéraires - car nous ne pourrions pas les géoréférencer de la même manière. La logique de la base est, en effet, résolument topographique. Néanmoins, nous avons tenu à intégrer les références littéraires, qui s'avèrent indispensables pour mener une réflexion sur le fait religieux, pour chaque lieu de culte, afin de proposer un panorama complet des sources. En revanche, nous avons temporairement mis de côté les nécropoles - extrêmement nombreuses -, afin d'éviter de ralentir la phase de dépouillement des chroniques de fouilles et de déséquilibrer l'identité de la base.

dell'Italia antica. 2. Regio I, Abella, Abellinum, Salerne. La publication des fascicules doit se poursuivre dans les années 2010-2013.

8. Cet inventaire est placé sous la direction de Meriem Sebai.

9. Séminaire intitulé : «Constructions, interprétations et représentations religieuses de l'espace ». 
Nous avons également été très attentifs à la terminologie des catégories de classement concernant les aménagements cultuels, les objets ou mobiliers trouvés en contexte, afin de ne pas perpétuer des interprétations hâtives ou abusives autour de termes très connotés, comme « offrande » ou « sacrifice », qui contribuent souvent à fausser l'analyse des vestiges. Nous avons aussi cherché à éviter les écueils d'un classement hiérarchique, tant au niveau des divinités (avec, par exemple, des appellations comme divinité principale et/ou secondaire), qu'au niveau des structures (temple, autel ou bâtiment), que l'on a choisi de ne pas présenter de manière «hiérarchisée» en fonction de leur importance supposée. Pour identifier sans interpréter, pour répertorier sans surdimensionner un objet, une structure, ou l'attribution d'un lieu de culte à une divinité. Cette réflexion critique sur les champs et les listes d'autorité de la base de données a permis de créer une architecture volontairement neutre, tout en affichant une description précise des éléments trouvés en contexte associée à des renvois bibliographiques précis et actualisables.

Pour renseigner la base, nous avons croisé plusieurs types d'informations en fonctionnant par strates successives de dépouillement. Ainsi, nous avons intégré les données des ouvrages et des colloques majeurs sur la Béotie et la topographie religieuse, en y associant les informations essentielles des chroniques de fouilles ${ }^{10}$ et des corpus épigraphiques ${ }^{11}$ systématiquement dépouillés. Les chroniques archéologiques nous ont permis de pouvoir afficher la situation exacte du lieu de culte, ses attributions successives, la complexité de sa datation, l'historique des fouilles, les structures, les objets trouvés en contexte, etc. Et le dépouillement épigraphique nous a permis de pouvoir rassembler toutes les inscriptions rattachées à un lieu de culte ou à une localité. Restait à organiser cette documentation. Effectivement, la prolifération des découvertes archéologiques donne un matériel riche, souvent contradictoire, mais surtout fragmenté. L'ambition de la base était donc aussi de rassembler de manière cohérente toutes ces données, de les présenter de manière précise et unifiée, mais aussi de les mettre en contexte, grâce à la carte générale articulée à des plans plus précis des localités ou même des lieux de culte, pour

10. Provenant notamment des revues majeures Archaiologikon Deltion, Bulletin de correspondance hellénique, Kernos, Chiron, Archaeological Reports, Hesperia, Zeitschrift für Papyrologie und Epigraphik.

11. $I G, S E G, B E$. 
pouvoir impulser des recherches de micro-topographie. En choisissant de ne pas présenter une multitude de cartes partielles associées individuellement à des fiches mais une carte générale, la base de données CIRCE affichera l'ensemble de la documentation dans une vision diachronique. On espère ainsi qu'elle fournira un outil pertinent pour " penser » la plasticité du paysage religieux, notamment grâce à un système de "calques » chronologiques qui permettront non seulement d'accéder à une vision précise du territoire religieux à une période donnée, mais aussi, à terme, de pouvoir mesurer l'évolution de l'occupation des lieux de culte à travers les siècles.

En effet, la base de données étant inscrite en permanence sur une carte, elle permet de territorialiser les données et de ne jamais séparer des informations de leur contexte topographique et religieux, ni même des réalités naturelles. Ce dépouillement, qui a amené la constitution des «notices » des lieux de culte, s'est ainsi doublé d'un travail de localisation grâce aux coordonnées géographiques la base de données relationnelle élaborée en Open Source étant articulée au système de référencement et de cartographie universels de Google Earth et Google Maps. Chaque site est ainsi localisé avec précision par son toponyme actuel géoréférencé associé à sa localisation antique. Quand les données nous le permettent, nous affichons le géoréférencement du lieu de culte lui-même. Chaque lieu de culte est donc à la fois dépouillé dans une notice, mais également identifié et localisé précisément sur la carte générale. Ce double affichage des informations permet ainsi une approche des sites répertoriés par navigation sur les vues satellitaires ou sur les cartes, et un accès par le tableau des notices des lieux de culte répertoriés.

La base de données, mise en ligne et associée à un système d'information géographique, recense donc actuellement les divers lieux de culte de Béotie, géoréférencés et présentés de manière raisonnée sous forme de fiches regroupant les données qui leur sont relatives. Tout d'abord, la notice affiche la localisation toponymique (moderne et ancienne), une description du lieu de culte associée à une bibliographie générale et à un historique des fouilles, avec des liens vers les chroniques archéologiques en ligne (type CEFAEL ${ }^{12}$ ).

12. La bibliothèque numérique regroupant l'intégralité des ouvrages publiés par l'École française d'Athènes depuis 1877 et notamment le Bulletin de correspondance hellénique. 
Pour l'avenir, l'ambition est de multiplier ces liens avec d'autres centres de ressources numériques. Puis, elle dépouille les données au travers de deux catégories reliées chacune à des listes d'autorité, « structure $^{13}$ » et « mobiliers ${ }^{14}$ ». À travers ces deux classifications, la base permet de pouvoir affiner les informations sur ces structures et ces mobiliers avec, pour chaque recension, un large champ permettant la description, les références bibliographiques et littéraires, une bibliographie scientifique actualisée issue notamment du dépouillement systématique des chroniques de fouilles, une indication chronologique par période et/ou par siècle ${ }^{15}$, les indications épigraphiques précises (lorsque le mobilier porte une inscription avec le type d'inscription ${ }^{16}$ ), la référence d'édition et le texte épigraphique en Unicode ${ }^{17}$. Le programme vise également à élaborer une véritable planimétrie des lieux de culte : il est effectivement prévu que l'on puisse intégrer des plans et des photographies du site et/ou des monuments, afin d'unifier les plans déjà accessibles dans les chroniques de fouilles proposées en lien.

Les champs de la base sont donc volontairement larges, tant au niveau des «structures » que du «mobilier» trouvé en contexte. Pourtant, malgré ce parti pris, nous avons la possibilité de mentionner pour chaque recension les interprétations contradictoires et d'actualiser les données, afin que les consultants puissent accéder rapidement à toutes les sources et informations bibliographiques actuellement disponibles sur ce sujet. Si l'inventaire ne peut évi-

13. Choix possibles dans la liste d'autorité «Structure»: Temple/Autel/ Péribole/Bâtiment/Portique/Fontaine/Bains/Théâtre/Autre.

14. Choix possibles dans la liste d'autorité « Mobilier»: Statue/Base de statue/Statuette-figurine/Monnaie/Objet personnel/Arme/Trépied/Vase (céramique ou autre)/Ossements-restes organiques/Relief/Stèle/Pinax/Autre.

15. Choix possibles dans la liste d'autorité «Chronologie»: Helladique Récent (HR) I.II.IIIA-B/HR IIIC/Sub-Mycénien/Proto Géométrique/Géométrique (Géométrique Ancien, Géométrique Moyen, Géométrique récent)/Archaïque (VII ${ }^{\mathrm{e}}$ s.-VI ${ }^{\mathrm{e}} \mathrm{s}$.)/Classique $\left(\mathrm{V}^{\mathrm{e}} \mathrm{s}\right.$.-IV $\mathrm{IV}^{\mathrm{e}} \mathrm{s}$.)/Hellénistique $\left(\mathrm{III}^{\mathrm{e}} \mathrm{s}\right.$.) $/ \mathrm{II}^{\mathrm{e}} \mathrm{s}$.// $\mathrm{I}^{\mathrm{er}} \mathrm{s}$. av. J.-C./ Époque romaine $\left(\mathrm{I}^{\mathrm{er}}\right.$ s. ap. J.-C./ $\mathrm{II}^{\mathrm{e}}$ s. apr. J.-C./ $\mathrm{III}^{\mathrm{e}}$ s. apr. J.-C./ IV ${ }^{\mathrm{e}}$ s. apr. J.-C./- $\mathrm{v}^{\mathrm{e}}$ s. apr. J.-C.). Il existe également la possibilité de mentionner un terminus post quem/terminus ante quem.

16. Choix possibles dans la liste d'autorité «Type inscription »: Dédicace/ Règlement cultuel/Décret/Inscription honorifique/Autre.

17. Pour accéder à des informations plus précises, nous avons créé une deuxième page qui permet de mentionner des données typologiques et un numéro d'inventaire pour un objet conservé dans un musée. Nous avons aussi créé la possibilité de mentionner une « quantité » chiffrée complétée par des opérateurs pour la prise en compte de quantités approximatives $(>,<,=\ldots)$. 
demment livrer tout le détail des informations, il réunit et donne une vue d'ensemble à jour de sources documentaires de natures très différentes (depuis les aménagements monumentaux jusqu'aux restes organiques, en passant par les inscriptions, les sources littéraires et les traces des rites accomplis). Cela dans une perspective chronologique large, visant à proposer une vision synoptique de la "vie» des lieux de culte dans leur contexte spatial, institutionnel et social. Grâce à sa grande souplesse, la base est évolutive en tous points. L'on pourra donc à loisir rajouter des précisions supplémentaires, l'actualiser, l'enrichir ou la complexifier. Plusieurs niveaux d'accès sont en cours de finalisation afin de permettre à la fois le travail en réseau pour les membres du groupe de recherche et une consultation large, tout en préservant la confidentialité des données ${ }^{18}$.

\section{RÉSUltats, POSSIBILITÉS DE RECHERCHE ET DÉVELOPPEMENT DE LA BASE}

À ce jour, la base a géoréférencé 31122 toponymes, établi 136 notices « lieu de culte », propose 620 fiches «structures »/ «mobiliers » et plus de 1000 références bibliographiques. L'outil actuellement en ligne va évoluer, en passant du stade d'une base de données à un support multibases intitulé Open melodie. Cette plateforme logicielle en Open source, en cours d'élaboration au Collège de France, doit permettre la cohabitation et la gestion en ligne de plusieurs bases de données comme le MAVI (Musée Achéménide Virtuel et Interactif) et le projet CIRCE. Mais la base CIRCE permet, d'ores et déjà, plusieurs possibilités d'interrogation croisées. Il est, en effet, possible de faire des recherches à partir de tous les niveaux de la base (chercher un toponyme, un lieu de culte, une structure ou un mobilier), mais également dans tous ses champs (description de la structure ou du mobilier, type d'inscription, référence épigraphique, informations relatives aux figures divines, datation...).

18. Un premier niveau de " consultation » est ouvert à tous les utilisateurs avec des restrictions concernant notamment les coordonnées géographiques des lieux de culte. Le niveau «saisie/modification» est accordé aux membres du groupe de recherche qui renseignent la base et peuvent ajouter des informations supplémentaires, voire modifier les données d'une fiche existante après validation par le modérateur. 
Naturellement, on peut aussi croiser plusieurs critères pour effectuer une recherche complexe : tous les temples consacrés à une divinité, les dédicaces concernant un dieu, les mentions d'une épiclèse à une époque donnée, les lieux de culte associant un temple et un autel, etc. La page de résultat, construite sous la forme d'un tableau raisonné, permet ensuite d'accéder à chacune des notices pour consulter les informations détaillées, mais également pour replacer ces informations dans leur contexte topographique et religieux. Des fonctionnalités de recherche supplémentaires sont en cours de réalisation, en particulier la prise en compte des critères topographiques (situation géographique précise, proximité avec différentes réalités naturelles). L'ambition est d'afficher plus systématiquement les réponses des recherches sur la carte générale.

Mais, la base de données n'est pas une fin en soi, car le projet reflète et nourrit une réflexion plus large sur l'espace religieux, qui a déjà abouti à l'organisation d'une Journée d'étude en $2008^{19}$, à des présentations successives à l'université de Fribourg, lors de la Troisième rencontre archéologique sur la Thessalie et la Grèce Centrale à Volos ${ }^{20}$, au Deutsch-Institut de Berlin ${ }^{21}$, au colloque de bilan de l'ANR ${ }^{22}$, à l'École française d'Athènes en vue de finaliser un partenariat de recherche ${ }^{23}$, et au XII ${ }^{\mathrm{e}}$ colloque du CIERGA ${ }^{24}$. Enfin, le projet s'est incarné dans le colloque international intitulé «Qu'est-ce qu'un paysage religieux? », qui s'est tenu à l'INHA et au Collège de France les 8-9 avril 2009. Si la base classe inévitablement les données selon des catégories et peut, de ce fait, donner l'impression de figer les informations, la fonction " recherche » a été conçue pour déconstruire cet apparent classement afin de présenter les traces matérielles de la présence d'une divinité sur le territoire.

19. Journée d'étude « CIRCE. Méthode et questionnements de l'inventaire des lieux de culte $»$ (INHA, 5 avril 2008).

20. $3^{\mathrm{e}}$ AETHSE, 12 au 12 mars 2009.

21. Dans le cadre du séminaire «Religiöse Landschaft Griechenlands » (Université de Fribourg/Allemagne, 3 décembre 2008).

22. 24 février 2009.

23. Sonia Darthou : «La base de données CIRCE : principes méthodologiques et présentation de résultats ", septembre 2009.

24. Colloque Archéologie et religion grecque: nouvelles découvertes, nouvelles perspectives et diffusion de l'information, Dion, 24 au 24 septembre 2009. Sonia Darthou, "Présentation thématique et méthodologique du programme CIRCE 》. 
Le projet CIRCE, qui articule un outil informatique avec des problématiques de recherches, permettra, nous le souhaitons, de mieux appréhender la topographie religieuse de l'Antiquité et d'éclairer des thèmes actuels de recherche : ainsi la notion de l' " occupation religieuse du territoire $»$, les questionnements sur la nature et la définition d'un lieu de culte, ou les liens entre réalités naturelles et lieux de culte.

UMR 8210 ANHIMA

2 rue Vivienne

75002 Paris

soniadarthou@yahoo.fr 